\title{
Seguridad y salud en el trabajo en Colombia: retos frente a las personas con discapacidad*
}

\author{
Safety and health at work in Colombia: challenges against persons with disabilities
}

\section{Resumen}

De acuerdo a los informes de las organizaciones multilaterales y nacionales el número de personas con discapacidad $(\mathrm{PcD})$ aumentan progresivamente en el mundo y una esfera importante en la cual desarrollan sus capacidades es el trabajo. Por lo tanto, los Sistemas de Gestión de Seguridad y Salud en el Trabajo deben preocuparse por su integración y el cumplimiento eficaz de las normas que buscan garantizar condiciones dignas en los ambientes de trabajo. Los retos actuales implican integrar adecuadamente el ciclo PHVA, las normas laborales y aplicar los ajustes razonables en el trabajo.

Natalia Eugenia Gómez Rúa**

${ }^{* \star}$ Abogada. Magister en Derecho. Especialista en Derecho de la Seguridad Social. Estudiante del Doctorado en Salud Pública de la Universidad CES. Coordinadora de Postgrados de la Facultad de Derecho de la Universidad CES. Investigadora del Observatorio en Salud Pública y Grupo de Estudios Jurídicos de la Universidad CES, reconocidos por COLCIENCIAS

Correo electrónico: ngomez@ces.edu.co

Felipe Turizo Peláez*** ${ }^{* * *}$ Abogado. Especialista en Gerencia en Seguridad Social. Asesor Jurídico de Hospitales. Integrante del Grupo de
Estudios Jurídicos de la Universidad CES Correo electrónico: pipeturizo@gmail.com

Palabras clave: Riesgos laborales, OIT, normatividad, PVHA, ajustes razonables.

\begin{abstract}
According to the reports of the multilateral and national organizations, the number of people with disabilities $(\mathrm{PwD})$ have increased progressively in the world and the major area in which they develop their skills is in work.

Therefore, Occupational Health and Safety Management Systems should be concerned with their integration and effective compliance with standards that seek to ensure and dignified the conditions in the workplace. The current challenges involve properly integrating the PDCA cycle, labor standards and applying reasonable adjustments in work.
\end{abstract}

Keywords: Ocupacional hazards, ILO, regulations, PDCA, reasonable adjustments.

\section{Introducción}

La relevancia de abordar desde la esfera jurídica y social la discapacidad es innegable ante los datos suministrados por la Organización Mundial de la Salud (OMS) en la Encuesta Mundial de Salud y los reportes de la Organización Internacional del Trabajo sobre las tasas de desempleo de las PcD(ILO International Programme on Safety and Health at Work and the Environment, 2011; International Labour Office, 2002). La OMS afirma que "En el mundo hay más de 1000 millones de personas con discapacidad, lo que supone alrededor de un $15 \%$ de la población mundial. De todas ellas, entre 110 y 190 millones de adultos padecen dificultades funcionales importantes."(OMS, 2014)

"Este artículo es producto de las investigaciones "Integración de las personas con discapacidad en los sistemas de gestión de seguridad y salud en el trabajo en los debates legislativos de la legislación Colombiana: Análisis normativo 1997 - 2014" y "Aplicación de la línea jurisprudencial sobre estabilidad laboral reforzada por discapacidad - el caso del Consultorio Jurídico de la Universidad CES entre 2011-2016"inscritos a la Línea de Investigación "Derecho Laboral, Seguridad Social y Responsabilidad Médica" del Grupo de Estudios Jurídicos de Facultad de Derecho de la Universidad CES. 
A partir del marco internacional y los convenios ratificados por Colombia se han creado parámetros de protección a las personas con discapacidad, tanto en la Constitución Política como en normas como las leyes 361 de 1997 y 1618 de 2013. Concretamente en materia de Riesgos Laborales, se ha desarrollado un referente básico fundamentado en la Ley 1562 de 2012 y en el Decreto 1072 de 2015 que incluye el Sistema de Gestión de Seguridad y Salud en el Trabajo (SG-SST), en el cual tiene como finalidad el bienestar del trabajador, incluyendo a las personas con discapacidad.

En este artículo, se analizará la evolución normativa en riesgos laborales, la relación del concepto de trabajo en relación con las $\mathrm{PcD}$, la implementación del SG-SST y por último la estrategia de ajustes razonables como un asunto de la seguridad y salud para los trabajadores con discapacidad.

La pretensión de este texto es aportar elementos para las futuras regulaciones normativas que se requieren en el país para lograr la vinculación laboral inclusiva y que las empresas puedan visualizar estrategias para la aplicación del Sistema de Gestión de Seguridad y Salud en el Trabajo respecto a las Personas con Discapacidad.

\section{Evolución normativa en Riesgos Laborales}

Colombia, como estado miembro de la Organización Internacional del Trabajo (OIT), ha ratificado diferentes convenios tendientes a garantizar la protección de los trabajadores y prestaciones económicas derivadas de accidentes y enfermedades laborales. De estas normas internacionales sobresalen las siguientes: 161 sobre servicios de salud en el trabajo, 13 sobre la cerusa (pintura con plomo), 18 sobre las enfermedades profesionales, 81 sobre la inspección del trabajo, 136 sobre el benceno, 162 sobre el asbesto, 167 sobre la seguridad y salud en la construcción, 170 sobre los productos químicos y C174, sobre la prevención de accidentes industriales mayores.
En 1904, la normatividad interna previó la necesidad de pensar en la salud de los trabajadores. En esta época, Rafael Uribe, señaló que se requería dictar leyes sobre accidentes de trabajo, de las mujeres en los talleres y en los trabajos del campo y obligar a los patronos a preocuparse de la higiene, el bienestar y de la instrucción gratuita de los desamparados (Lizarazo, Fajardo, Berrio, \& Quintana, 2011). Este propósito se vio plasmado a través de las Ley 57 (Congreso de la República de Colombia, 1915), referida a las reparaciones en accidentes de trabajo.

Posteriormente, se gestaron otras específicas sobre la materia, como la Ley 9 de 1979 (Congreso de la República de Colombia, 1979, p. 9), en la que se reconoce que la salud de los trabajadores trasciende la relación patrono y trabajador y se estima que hace parte del desarrollo socioeconómico del país en la que deben participar gobiernos y particulares. Posteriormente, se crea la Ley 6 (Congreso de la República de Colombia, 1945) para los servidores públicos y el Código Sustantivo del Trabajo (Congreso de la República de Colombia, 1990) para los trabajadores particulares, en ambas se incluye el tema de riesgos profesionales y se determina el pago de prestaciones económicas derivadas de accidentes y enfermedades laborales.

Esta obligación patronal estuvo vigente hasta el Acuerdo 241 de 1967 (Instituto de los Seguros Sociales, 1967) que reglamentó la prevención de riesgos profesionales a cargo del Instituto Colombiano de Seguros Sociales (hoy liquidado), con la obligación para los empleadores de realizar aportes a esta entidad para cubrir las respetivas contingencias. Luego, se promulgó Ley 100 de 1993 (Congreso de la República de Colombia, 1993), que contempló el Sistema General de Riesgos Profesionales, reglamentado a través del Decreto 1295 de 1994. Posteriormente, se expidió la Ley 776 de 2002. En el marco normativo de Riesgos Profesionales siempre se pretendió atender dos 
componentes: la prevención y las prestaciones económicas, pero debe reconocerse que siempre se dio más énfasis a la parte prestacional que a prevenir los accidentes y las enfermedades en el trabajo.

Debido a los compromisos internacionales con la OIT y la preocupación frente al aumento de discapacidades por accidentes y enfermedades laborales en el mundo (OIT \& OMS, 2005), Colombia en el año 2012 expidió la Ley 1562(Congreso de la República de Colombia, 2012), a través de la cual se adecuó la terminología en prevención, de acuerdo a las indicaciones de la citada Organización, para atender las necesidades de los trabajadores a nivel global.

Concretamente se amplió el término programa de Salud Ocupacional por Sistema de Gestión de Seguridad y Salud en el trabajo con la finalidad de adecuar el bienestar del trabajador, en procesos y seguimientos. Se cambió la denominación de riesgos "profesionales" por "laborales" que incluye todas las actividades de trabajo. Por otra parte, se atiende las recomendaciones orientadas a que "una política nacional en materia de SST proporciona un marco de acción para garantizar que se minimicen en la medida en que sea razonable y factible las causas de los riesgos existentes en el medio ambiente de trabajo nacional." (International Labour Office \& International Labour Standards Department, 2013).

Actualmente, se ha gestado el desarrollo reglamentario para ejecutar el Sistema de Gestión en este campo como un tema laboral incluido en el Decreto Único del Sector Trabajo, 1072 de 2015(Presidencia de la República, 2015), no obstante su aplicación se encuentra prorrogada por el Decreto 171 para el año 2017 (Congreso de la República de Colombia, 2016). Es importante reconocer que esto implica adecuaciones a los empleadores, no solamente desde la seguridad y salud sino toda la plataforma laboral como revisión de políticas y reglamento interno de trabajo para que sea coherente con la planeación y ejecución de un Sistema de Gestión.

Dentro de esta planeación y ejecución, debe tenerse en cuenta a un grupo poblacional, tradicionalmente vulnerado en el ámbito laboral, las personas con discapacidad. Algunos estudios a nivel internacional advierten que representan las tasas más bajas de empleo dentro del colectivo y perciben menos salario (Navarro, Guerrero, \& Viana, 2015) y tienen peores condiciones de trabajo y salud (OMS, 2014).

\section{El trabajo y las personas con discapacidad}

Sobre el concepto de trabajo, se encuentra que ha sido abordado desde diferentes áreas, el diccionario de la real académica española de la lengua lo define como una ocupación retribuida(RAE, 2016). La OIT precisa que es "el conjunto de actividades humanas, remuneradas o no, que producen bienes 0 servicios en una economía, o que satisfacen las necesidades de una comunidad o proveen los medios de sustento necesarios para los individuos. De este concepto se desprende, el término empleo que se refiere concretamente al "trabajo efectuado a cambio de pago (salario, sueldo, comisiones, propinas, pagos a destajo o pagos en especie)" sin importar la relación de dependencia (si es empleo dependiente-asalariado, o independienteautoempleo)."(OMS, 2004)

En Colombia, el artículo $5^{\circ}$ del Código Sustantivo del Trabajo opta por la siguiente definición de trabajo: "Toda actividad humana libre, ya sea material o intelectual, permanente o transitoria, que una persona natural ejecuta conscientemente al servicio de otra, y cualquiera que sea su finalidad, siempre que se efectúe en ejecución de un contrato de trabajo"(Congreso de la República de Colombia, 1990)

Desde la perspectiva de la dignidad humana, hoy se habla de trabajo decente y se entiende 
como tal "la oportunidad de acceder a un empleo productivo que genere un ingreso justo, la seguridad en el lugar de trabajo y la protección social para las familias, mejores perspectivas de desarrollo personal e integración social, libertad para que los individuos expresen sus opiniones, se organicen y participen en las decisiones que afectan sus vidas, la igualdad de oportunidades y trato para todos, mujeres y hombres." (OIT, 2016)

Desde esta mirada, el trabajo decente es un derecho de todas las personas, pero en especial se debe proteger a ciertos grupos vulnerables como las personas con discapacidad. Sobre el concepto de PcD, la Convención de los Derechos de las Personas con Discapacidad (ONU, 2008), ratificada por Colombia, es un referente teórico actual para afirmar ha evolucionado. De éste se desprenden dos aspectos, el primero corresponde a la parte biológica y el segundo a la parte social, ya que define la discapacidad en la persona como "la interacción entre sus deficiencias físicas, psíquicas o sensoriales y las barreras que se les impone en la sociedad para la igualdad de condiciones y participación, debido a la actitud de los demás seres humanos en su entorno" (ONU, 2008).

En este sentido, la Organización Internacional del Trabajo (OIT), promueve para este grupo poblacional dentro de la agenda del desarrollo global, la Gestión de las discapacidades en el lugar de trabajo como "una herramienta para ayudar a los países miembros a poner en práctica las disposiciones de las normas del trabajo de la OIT pertinentes, el repertorio de Recomendaciones Prácticas de la OIT sobre la gestión de las discapacidades en el lugar de trabajo y la citada Convención. (International Labour Office, 2015). Entre los objetivos del repertorio de gestión de las discapacidades en el lugar de trabajo, se encuentran proporcionar orientaciones prácticas para asegurar que las personas con discapacidades tengan igualdad de oportunidades, mejorar las perspectivas de empleo, promover un lugar de trabajo seguro, accesible y saludable(International Labour Office, 2015).

Referirse a trabajo decente para las personas con discapacidad implica para los empleadores plantear estrategias eficaces para la seguridad y salud en el trabajo, este tema en la legislación colombiana, aunque no es novedoso, si ha implicado en los últimos años un trabajo normativo con la finalidad de garantizar condiciones adecuadas dentro de los ambientes de trabajo.

Por otra parte, es importante reconocer la diversidad de $\mathrm{PcD}$, puede encontrarse grupos poblaciones distintos como aquellos que su discapacidad se generó por una deficiencia de nacimiento, una enfermedad de origen común, un accidente o enfermedad de origen laboral. Incluso algunos tendrán una calificación de pérdida de capacidad laboral y otros no. Estas características significan que sus barreras para la igualdad de condiciones y oportunidades, deben ser abordadas de diferentes maneras, incluso desde el espectro normativo.

No obstante, por el carácter dinámico de la discapacidad, existe para los empleadores la obligación de protegerlos en los ambientes de trabajo, independiente de las causas que le dieron origen, en el sentido que existe la obligación de garantizar ambientes saludables lo cual se logra a través de los Sistemas de Gestión Seguridad y Salud en el Trabajo.

Como aspectos normativos básicos, aplicables a cualquier personas con discapacidad, las empresas deben tener en cuenta que la ley 361 de 1997 (Congreso de la República de Colombia, 1997, p. 361) establece mecanismos de integración social para las personas con discapacidad, siendo importante resaltar la protección en caso de ser despedidas en razón de su limitación física, síquica o sensorial sin la autorización del Ministerio de Trabajo, lo cual se ha denominado estabilidad laboral reforzada. Esta norma, condiciona el despido a cumplir 
con este protocolo para garantizar que no se presente una discriminación y en caso de no cumplirse, el empleador debe pagar adicional a las prestaciones e indemnizaciones de Ley, 180 días de salario.

Por otra parte, se promulgó la Ley 1618 de 2013 en la cual se establecen disposiciones para garantizar el pleno ejercicio de los derechos de las personas con discapacidad(Congreso de la República de Colombia, 2013), concretamente respecto al empleo establece la importancia de condiciones dignas, algunas prebendas tributarias para empleadores y beneficios para la contratación estatal. Aunque es importante que se reglamente esta Ley, su efectividad se encuentra enmarcada desde un enfoque de derechos, en el compromiso de brindar "accesibilidad universal, diseño para todas las personas y los ajustes razonables” (Pérez, 2012)

Para algunos empleadores, estas dos normas constituyen un obstáculo para la contratación laboral, lo cual se puede verificar en el reciente informe de la Coalición colombiana por la Implementación de la Convención sobre los Derechos de las Personas con Discapacidad (ONU, 2008, p. 41). Específicamente, la desmotivación para vincular $\mathrm{PcD}$, se atribuye a la interpretación del artículo 26 de la Ley 361 de 1997 por parte de la Corte Constitucional, a través de la cual se extiende la protección a toda persona que presente una afectación en su estado de salud sea de manera temporal o permanente, frente a lo cual los empleadores ven imposible la terminación de sus contratos, aun cuando medie causales de justificación, por lo que los asesores laboralistas desaconsejan la contratación de personas con discapacidad, aunque existen incentivos tributarios para su contratación.

Como estrategia para incentivar la contratación de $\mathrm{PcD}$ en el país, el Banco Internacional de Desarrollo (BID) y el Ministerio de Trabajo han liderado un Programa Empresarial de Promoción Laboral para las Personas con Discapacidad, denominado Pacto de Productividad («Pacto de Productividad. Promoviendo la Inclusión Laboral de las personas con discapacidad», 2015), en el cual participan el gobierno, las empresas y la sociedad civil. Este programa pretende la contratación inclusiva y de acuerdo al informe presentado en el año 2015, 5657 personas se han beneficiado y 142 empresas se han comprometido con la contratación y capacitación para el empleo(«Pacto de Productividad. Promoviendo la Inclusión Laboral de las personas con discapacidad", 2015). No obstante, a la fecha no hay ningún reporte que evidencie el seguimiento que se ha realizado a las empresas para verificar las condiciones de trabajo en las que se encuentran desempeñando sus laborales y la forma como se integra esta política inclusiva a los Sistemas de Gestión de Seguridad y Salud en el Trabajo (SG-SST).

Al respecto, se considera necesario integrar a la política de inclusión laboral, el SG-SST como estrategia para cumplir las normas de riesgos laborales y protección legal para las personas con discapacidad desde una perspectiva positiva y productiva. Para lograrlo se requiere revisar en que consiste la implementación del sistema y la articulación que debe darse respecto a las PcD como se explica a continuación:

\section{Implementación del Sistema de Gestión de Seguridad y Salud en el Trabajo (SG-SST) y las Personas con Discapacidad.}

El Sistema de Gestión de la Seguridad y Salud en el Trabajo SG-SST consiste en "el desarrollo de un proceso lógico y por etapas, basado en la mejora, continua y que incluye la política, la organización, la planificación, la aplicación, la evaluación, la auditoría y las acciones de mejora con el objetivo de anticipar, reconocer, evaluar y controlar los riesgos que puedan afectar la seguridad y la salud en el trabajo."(Congreso de la República de Colombia, 2012)

En el Decreto 1072 (Presidencia de la República, 2015), los ciclos en que debe surtirse 
este Sistema de Gestión, son definidos de la siguiente forma: "P" de planificar, es decir, las formas de mejorar la seguridad y la salud de los trabajadores, para saber que se está haciendo incorrectamente o que se debe mejorar, la "H" de hacer, lo cual es la implementación de las medidas planificadas, la " $\mathrm{V}$ " de verificar, que es revisar y analizar esas acciones implementadas en los pasos anteriores, y finalmente la " $A$ " de actuar que es realizar esas acciones para buscar siempre el mejoramiento en la seguridad y la salud de los trabajadores.

El Sistema de Gestión está basado en acciones que buscan la optimización de los procesos que lleven a las organizaciones a formar políticas coherentes para lograr mejorar tanto del punto de vista del empleador o contratante, y el trabajador o contratista todos aquellos factores que intervengan en el desarrollo de un adecuado trabajo, logrando mitigar todos los riesgos que puedan afectar la Seguridad y la Salud en el Trabajo (Presidencia de la República, 2015).

Además, en la citada norma (Presidencia de la República, 2015), se ha indicado que dicho sistema debe ser liderado e implementado por el empleador o contratante, con la participación de los trabajadores y/o contratistas, garantizando a través de él, la aplicación de las medidas de Seguridad y Salud en el Trabajo, el mejoramiento del comportamiento de los trabajadores, las condiciones y el medio ambiente laboral, y el control eficaz de los riesgos con el fin de prevenir los accidentes y las enfermedades laborales, así como también crear un ambiente de protección y prevención de estos eventos.

En el Decreto 1072 de 2015, es clara la intención del legislador de cuidar la salud de los trabajadores, por lo cual se exige la integración de un adecuado sistema de gestión en el marco del ciclo PHVA, con la finalidad de apoyarse en acciones de mejora y un adecuado control y manejo de riesgos, con indicadores, medidas de prevención y control.
Aunque no se visualiza en la ejecución del Sistema de Gestión la forma de integrar a las personas con discapacidad, si hay un margen normativo en el país que permitiría su adaptación e integración en entendido que este sistema debe comprender que los trabajadores pueden estar en situación de discapacidad de forma temporal o permanente. Al respecto se encuentran las siguientes Leyes, Decretos y Resoluciones:

El artículo 125 de la Ley 9 (Congreso de la República de Colombia, 1979), expone la obligación legal del empleador de implementar programas de medicina preventiva que tienen como objeto la rehabilitación y recuperación del trabajador. Posteriormente, a través de la Resolución 1016 (Resolución 1016, 1989) se crea la obligación del subprograma de medicina preventiva y del trabajo, que adicional a la rehabilitación, la reubicación de las personas con incapacidad temporal y permanente parcial. Esta parte normativa, aplica a cualquier trabajador que presente un evento de salud en el trabajo, pero implica un mayor compromiso con las PcD que adquieran una deficiencia física, psíquica o sensorial en el trabajo.

La citada Ley 361 (Congreso de la República de Colombia, 1997) en sus artículos 22 al 34, se refiere a la integración laboral de las personas en situación de discapacidad, en donde se estipulan además de políticas de empleo y de estudios, incentivos para los empleadores que contraten personas con discapacidad. Al respecto, la norma aplica a cualquier trabajador con discapacidad sin importar su origen.

Luego, el artículo $4^{\circ}$ de la Ley 776 , prevé que luego de "terminar el período de incapacidad temporal, los empleadores están obligados, si el trabajador recupera su capacidad de trabajo, a ubicarlo en el cargo que desempeñaba, o a reubicarlo en cualquier otro para el cual esté capacitado, de la misma categoría. (Congreso de la República de Colombia, 2002). Esta norma también aplica para eventos ocurridos alrededor del trabajo. 
Por su parte, el Decreto 1072 de 2015, impone obligaciones a las entidades administradoras de riesgos laborales respecto a los servicios de rehabilitación de sus afiliados y de evaluación de los resultados de los programas de rehabilitación de la salud de los trabajadores, esto a través de los indicadores para identificar la efectividad de estos programas de rehabilitación. (Presidencia de la República, 2015). Sobresale de esta norma la necesidad de integrar a cualquier persona con discapacidad sin importa la causa que da lugar a las barreras para la igualdad de oportunidades y participación.

Otros asuntos debatidos en el Decreto Único del Sector Trabajo, en materia de Seguridad Salud en el Trabajo que atañen a las Personas con Discapacidad, son aquellos referidos a la reinserción laboral. Por ejemplo, la obligación de los sistemas de información del servicio público de empleo para la capacitación y la colocación respecto a la reinserción laboral, lo relacionado a las prestaciones económicas a la población cesante y la capacitación para la reinserción a través de los programas de capacitación para el empleo en condiciones dignas (Presidencia de la República, 2015).

No obstante ser claros los propósitos del SG-SST que aplican para las $\mathrm{PcD}$, la pregunta que debe realizarse en el marco del Sistema de Riesgos Laborales, es ¿cómo se garantiza a las personas con discapacidad el acceso, permanencia y condiciones dignas de trabajo? Lo anterior teniendo en cuenta que se han considerado que son elementos fundamentales para lograr la inclusión laboral de esta población (Zondek , Andrea, 2015).

Al respecto, se estima que solamente es posible garantizar a las PCD una verdadera inclusión desde el concepto de empleo digno en el marco de los SG-SST, cumpliendo con los ajustes razonables contemplados en la citada Convención y definidos como "unas medidas específicas que tienen como objeto o meta la accesibilidad en casos particulares, pero que se adoptan cuando ésta no es posible desde la previsión del diseño para todos, teniendo en consideración las necesidades específicas de una persona" (Palacios, 2008).

El tema de ajustes razonables, aunque tiene un amplio desarrollo en el ámbito internacional en nuestra legislación apenas se encuentra referenciado en la citada Ley 1618 de 2013 y todavía no ha sido reglamentado, lo que impide su obligatoriedad y cumplimiento en los entornos de trabajo. De forma adversa se ha considerado que su implementación es costosa, no obstante, se ha logrado desvirtuar esta premisa ya que algunos estudios evidencian que "generalmente suelen ser sencillos y de bajo costo. Uno de los criterios que parecen más sólidos para considerar la "razonabilidad" del ajuste en el puesto de trabajo, es el criterio económico" (OIT et al., 2015) como se explica en el siguiente acápite.

\section{Ajustes razonables para las personas con discapacidad, un asunto del SG-SST.}

En cuanto al entorno laboral, los ajustes razonables pretenden la igualdad de condiciones de las personas con discapacidad, lo cual implica las adecuaciones necesarias en el puesto de trabajo (Salazar, 2016). De forma acertada Pérez (Pérez, 2012), advierte desde el punto de vista jurídico los ajustes razonables requieren una reglamentación para evitar una inseguridad jurídica propia de los conceptos abiertos.

Al respecto el Comité sobre los Derechos de las Personas con Discapacidad, en el informe de agosto de 2016, manifiesta su preocupación sobre la ausencia de una regulación en la materia y solicita al Estado adoptar normas que traten este aspecto en materia de empleo (Comité sobre los Derechos de las Personas con Discapacidad, 2016)

En materia de regulación normativa, es importante tener en cuenta aspectos considerados en legislaciones foráneas, en las cuales se ha precisado que los ajustes razonables, aunque 
tiene como fines permitir a las personas con discapacidad acceder al empleo, desempeñar su trabajo, progresar profesionalmente y acceder a la formación, no pueden convertirse en medidas que supongan una carga excesiva para el empresario. En esta línea se ha revisado la importancia de las ayudas públicas, los costos de la adaptación de puestos de trabajo y el tamaño de las empresas. (Colominas, 2015). En el caso de Colombia esto implica una adecuación de estos criterios a las realidades sociopolíticas que permitan una normatividad que no sea solamente creada, sino que sea eficaz, es decir, se pueda aplicar. En este sentido se deberá tener en cuenta lo siguiente:

Respecto a ayudas públicas, es importante revisar los programas a nivel regional que se ofrecen en los municipios para la capacitación laboral, en Medellín ha liderado esta línea el proyecto "Ser Capaz" de la Alcaldía siendo importante revisar el compromiso de las empresas privadas a través de otras estrategias como el citado Pacto de Productividad («Pacto de Productividad. Promoviendo la Inclusión Laboral de las personas con discapacidad», 2015). No obstante, también deben destinarse recursos para los empleadores comprometidos con el trabajo inclusivo adicionales a los beneficios tributarios que actualmente se consagran, como se viene haciendo en otros países como Chile, España y Canadá.

Con relación a los costos de adaptación de los puestos de trabajo, es indiscutible que se requiere de una adecuación de espacios físicos y los costos económicos inicialmente pueden ser representativos, para adquirir, adecuar equipos o cambios estructuras. "En el trabajo, la accesibilidad se refiere a la facilidad con que los trabajadores pueden utilizar los locales, permitiéndoles serlomás independientes posible. Esto es aplicable a todas las discapacidades, incluidas las de movilidad, aprendizaje, visuales 0 auditivas. Existen numerosas medidas básicas y económicas que pueden marcar una gran diferencia"(Agencia Europea para la Seguridad y Salud en el Trabajo, 2004)
Lo anterior implica otras estrategias como son horarios flexibles según la discapacidad, es decir, revisar el número de horas que puede laborar el trabajador(a), también se puede considerar la ubicación del puesto del trabajo para facilitar la accesibilidad según la infraestructura de la empresa, garantizar una iluminación adecuada e incluso acudir a otras modalidades para desempeñar actividades como el teletrabajo, previendo los riesgos ocupacionales a los cuales puede estar expuesta la persona.

Sobre el tamaño de las empresas, puede plantearse una estrategia escalona, en la cual se vincule primero las grandes empresas y luego a las medianas y pequeñas empresas. En la medida que se evidencien fortalezas en la contratación de $\mathrm{PcD}$, se podrá documentar el costo-beneficio y las estrategias requeridas para que sean realmente razonables y no sea excesivo para quien contratan.

Luego de aplicar estos criterios, es necesario articular los ajustes razonables a la elaboración y ejecución de Seguridad y Salud en el Trabajo, para verificar su cumplimiento y el aporte al bienestar de los trabajadores. Una herramienta fundamental para lograrlo será la matriz legal(Presidencia de la República, 2015), la cual es de carácter obligatorio a partir de la implementación del Sistema e implica la descripción de lo que se requiere, evidencia de su cumplimiento y un responsable del mismo. También implica dar aplicación a las evaluaciones ocupacionales de preingreso y periódicas, a través de las cuales se podrá prever las recomendaciones y restricciones para cumplir satisfactoriamente las funciones.

Adicionalmente, en la política del SG-SST puede trazarse una estrategia general sobre inclusión, en la que necesariamente no solamente se hable de las personas con discapacidad, sino que de los grupos socialmente vulnerados, lo cual se articula con la misión, visión o programas de responsabilidad social de muchas empresas. 
Por último, es de advertir que estos retos son generales ya que la dinámica de su aplicación implicará un trabajo colectivo por parte del Estado, empleadores, asociaciones y de las propias $\mathrm{PcD}$, quienes se deben empoderar cada día más de sus derechos y sus obligaciones en materia de trabajo. Generar una cultura de empleo para $\mathrm{PcD}$ es un trabajo de todos que conlleva a un cuestionamiento proactivo en materia legislativa en aras de la regulación real de la Ley 1618 de 2013, los SG-SST y demás afines a la materia.

\section{A modo de conclusión}

De acuerdo a lo expuesto es posible concluir lo siguiente:

En Colombia se ha dado una evolución normatividad en materia de riesgos laborales que ha permitido llegar a establecer la necesidad de implementar un Sistema de Gestión de Seguridad y Salud en el trabajo, el cual respecto a las personas con discapacidad tiene el reto de su integración para garantizar condiciones dignas y disminuir las barreras para la participación y la igualdad.

La evolución del vocablo trabajo, ha permitido comprender en la actualidad su acepción de trabajo decente, basado en la construcción de derechos humanos, especialmente la dignidad. Desde esta óptica en los ambientes laborales debe existir una prioridad con los grupos considerados socialmente vulnerables como las personas con discapacidad.

Por último, se encuentra que se requiere la implementación de la normatividad existente en materia de Seguridad y Salud en el Trabajo, para garantizar a las PcD una integración real, siendo los ajustes razonables una estrategia importante para aplicar. No obstante, se requiere de una reglamentación donde expresamente se establezcan deberes y obligaciones con respecto a esta población.

\section{Referencias}

Agencia Europea para la Seguridad y Salud en el Trabajo. (2004). Garantizar la seguridad y la salud de los trabajadores con discapacidad. Recuperado a partir de https://www.healthy-workplaces. eu/sites/default/files/publications/documents/Factsheet 53 - Garantizar la seguridad_y la salud de los trabajadores con discapacidad.pdf

Comité sobre los Derechos de las Personas con Discapacidad. (2016, agosto 31). Convención sobre los derechos de las personas con discapacidad. Naciones Unidas. Recuperado a partir de http://www.ohchr.org/SP/NewsEvents/Pages/DisplayNews.aspx?NewsID=20389\&LangID=S

Congreso de la República de Colombia. Ley 57 de 1915 (1915). Recuperado a partir de http://www.alcaldiabogota.gov.co/sisjur/normas/Norma1.jsp?i=12998

Congreso de la República de Colombia. Ley 6 de 1945 (1945). Recuperado a partir de http://www.alcaldiabogota.gov.co/sisjur/ normas/Norma1.jsp?i=1167

Congreso de la República de Colombia. Ley 9 de 1979 (1979). Recuperado a partir de http://www.redlactea.org/wp-content/ uploads/decretos/L9.pdf

Congreso de la República de Colombia. Ley 50 de 1990 (1990). Recuperado a partir de http://www.secretariasenado.gov.co/senado/basedoc/codigo sustantivo trabajo.html

Congreso de la República de Colombia. Ley 100 de 1993, Pub. L. No. Diario Oficial No. 41.148 de 23 de diciembre (1993). Recuperado a partir de http://www.alcaldiabogota.gov.co/sisjur/normas/Norma1. isp?i=5248 
Congreso de la República de Colombia. ley 361 de 1997 (1997). Recuperado a partir de https://www.google.com.co/?gws $\underline{r d=s s \mid \# q=l e y+361+d e+1997}$

Congreso de la República de Colombia. Ley 776 de 2002 (2002). Recuperado a partir de http://www.mintrabajo.gov.co/normatividad-julio-decretos-2014/3700-decreto1443-del-31-de-julio-de-2014.html

Congreso de la República de Colombia. Ley 1562 de 2012 (2012). Recuperado a partir de http://wsp.presidencia.gov.co/Normativa/Leyes/Documents/ley156211072012. $\underline{\mathrm{pdf}}$

Congreso de la República de Colombia. Ley 1618 de 2013, Pub. L. No. Diario Oficial 48.717 de 27 de febrero de 2013 (2013). Recuperado a partir de http://www.bdigital.unal.edu.co/40045/

Congreso de la República de Colombia. Decreto 171 de 2016 (2016). Recuperado a partir de http://es.presidencia.gov.co/ normativa/normativa/DECRETO $\% 20$ 171\%20DEL\%2001\%20DE\%20FEBRERO\%20DE\%202016.pdf

ILO International Programme on Safety and Health at Work and the Environment. (2011). Sistema de gestión de la SST: una herramienta para la mejora continua : día mundial de la seguridad y la salud en el trabajo, 28 de abril 2011. Ginebra: OIT. Instituto de los Seguros Sociales. Acuerdo 241 de 1967 (1967).

International Labour Office. (2002). Gestión de las discapacidades en el lugar de trabajo: repertorio de recomendaciones prácticas de la OIT. Ginebra: OIT.

International Labour Office. (2015). Trabajo decente para las personas con discapacidad: promoviendo los derechos en la agenda mundial para el desarrollo. Ginebra: OIT.

International Labour Office, \& International Labour Standards Department. (2013). Crear una cultura de prevención en materia de seguridad y salud: guía sobre el Convenio sobre seguridad y salud de los trabajadores, 1981 (núm. 155), su Protocolo de 2002 y el Convenio sobre el marco promocional para la seguridad y salud en el trabajo, 2006 (núm. 187). Ginebra: OIT.

Lizarazo, C. G., Fajardo, J. M., Berrio, S., \& Quintana, L. (2011). Breve historia de la salud ocupacional en Colombia. Archivos de prevención de riesgos laborales, 14(1), 38-42.

Navarro, J. L., Guerrero, I., \& Viana, R. A. (2015). Economía laboral y discapacidad: reflexiones en torno a un asunto de salud pública. MedUNAB, 18(1), 71.

OIT. (2016). Trabajo decente. Recuperado 12 de agosto de 2016, a partir de http://www. ilo.org/global/topics/decent-work/lang-es/index.htm

OIT, \& OMS. (2005, abril 28). Comunicado conjunto OIT/OMS El número de accidentes y enfermedades relacionados con el trabajo sigue aumentando OIT y OMS recomiendan aplicar estrategias de prevención [Comunicado de prensa]. Recuperado 27 de julio de 2016, a partir de http://www.ilo.org/global/about-the-ilo/ newsroom/news/WCMS 006102/lang-es/index.htm

OIT, Red de Empresas Inclusivas Costa Rica, Asociación Empresarial para el DesarroIlo, United Way, Ministerio de Trabajo y Seguridad Social, \& PNUD. (2015). Caja de Herramientas Empresas Inclusivas (pp. 1-15). Costa Rica. Recuperado a 
partir de http://www.aedcr.com/redempresasinclusivas/files/cajaDeHerramientas/modulo 6.pdf

OMS. (2004). ¿Qué es el trabajo decente? [Noticia]. Recuperado 16 de agosto de 2016, a partir de http://www.ilo.org/americas/sala-de-prensa/WCMS_LIM 653 SP/lang--es/index.htm

OMS. (2014). Plan de acción mundial de la Organización Mundial de la Salud sobre discapacidad 2014-2021: mejor salud para todas las personas con discapacidad. Recuperado 13 de abril de 2015, a partir de http://apps.who.int/gb/ebwha/ pdf files/WHA67/A67 ACONF3-sp.pdf

ONU. (2008). Convención sobre los derechos de las personas con discapacidad. Naciones Unidas. Recuperado a partir de http:// joomla.corteidh.or.cr:8080/joomla/images/stories/Observaciones/2/Anexo\%20 XVII/L-8661\%20convenci\%C3\%B3n\%20 d\%20pe\%20con\%20discapa.doc

Pacto de Productividad. Promoviendo la Inclusión Laboral de las personas con discapacidad. (2015). Recuperado a partir de http://www. fundacioncoronamedios. org/pactodeproductividad2015/files/assets/common/downloads/publication.pdf

Palacios, A. (2008). Medidas relacionadas con la igualdad y la no discriminación en la implementación de la convención internacional sobre los derechos de las personas con discapacidad. Recuperado a partir de http://orff.uc3m.es/hand$\underline{\text { le/10016/9900 }}$
Pérez, L. C. P. (2012). La configuración jurídica de los ajustes razonables. En 20032012, 10 años de legislación sobre no discriminación de personas con discapacidad en España: estudios en homenaje a Miguel Ángel Cabra de Luna (pp. 159-183). Ediciones Cinca. Recuperado a partir de http://www.convenciondiscapacidad.es/Noticias/LA\%20CONFIGURACION\%20JURIDICA\%20DE\%20 LOS\%20AJUSTES\%20RAZONABLES. pdf

Presidencia de la República. Decreto 1072 de 2015 (2015). Recuperado a partir de http://www.fondoemprender.com/Normatividad/Decreto\%201072-2015.pdf

RAE. (2016). Real Academia Española. Recuperado 12 de agosto de 2016, a partir de http://www.rae.es/

Resolución 1016 (1989). Recuperado a partir de http://www.alcaldiabogota.gov.co/sisjur/normas/Norma1.jsp?i=5412

Salazar, E. R. B. (2016). La idea de los ajustes razonable como forma complementaria para conseguir la igualdad de las personas con discapacidad. pdf. Actualidad Jurídica-Universidad del Norte (Barranquilla, Colombia), 8, 40-54.

Zondek , Andrea. (2015). Manual para una inclusión laboral efectiva de personas con discapacidad. Chile: Servicio Nacional de Capacitación y Empleo. Recuperado a partir de http://www.senadis.gob.cl/ descarga/i/2874/documento

Forma de citar: Gómez, N., Turizo, F. (2016). Seguridad y salud en el trabajo en Colombia: retos frente a las personas con discapacidad. Rev. CES Derecho, 7(2), 84-94. 